\title{
Ferromagnetic transition temperature enhancement in (Ga,Mn)As semiconductors by carbon codoping
}

\author{
T. Jungwirth, ${ }^{1,2}$ J. Mašek, ${ }^{3}$ Jairo Sinova, ${ }^{2,4}$ and A. H. MacDonald ${ }^{2}$ \\ ${ }^{1}$ Institute of Physics ASCR, Cukrovarnická 10, 16253 Praha 6, Czech Republic \\ ${ }^{2}$ University of Texas at Austin, Physics Department, 1 University Station C1600, Austin, Texas 78712-0264, USA \\ ${ }^{3}$ Institute of Physics ASCR, Na Slovance 2, 18221 Praha 8, Czech Republic \\ ${ }^{4}$ Department of Physics, Texas A\&M University, College Station, Texas 77843-4242, USA \\ (Received 23 June 2003; revised manuscript received 13 August 2003; published 24 October 2003)
}

\begin{abstract}
We present a theoretical study of $(\mathrm{Ga}, \mathrm{Mn})(\mathrm{As}, \mathrm{C})$ diluted magnetic semiconductors with high $\mathrm{C}$ acceptor density that combines insights from phenomenological model and microscopic approaches. A tight-binding coherent-potential approximation is used to describe the electronic structure in the presence of $\mathrm{Mn}_{\mathrm{Ga}}$ and $\mathrm{C}_{\mathrm{As}}$ impurities. We find only a small effect of $\mathrm{C}$ on the distribution and coherence of electronic states close to the top of the valence band and on the coupling between Mn moments, even at doping levels of several per cent. These results justify applying the model of ferromagnetic Mn-Mn coupling mediated by itinerant holes in the valence band also to $\mathrm{C}$ doped samples. The increase of ferromagnetic transition temperature due to the presence of $\mathrm{C}$ acceptors is illustrated by calculations that use the $\mathbf{k} \cdot \mathbf{p}$ Kohn-Luttinger description of the GaAs valence band and assume systems where Mn local moment and itinerant hole densities can be varied independently.
\end{abstract}

DOI: 10.1103/PhysRevB.68.161202

PACS number(s): 75.50.Pp, 75.30.Et, 71.15.Nc

Prospects for new device functionalities in allsemiconductor spin-electronic structures rely on the realization of a ferromagnetic semiconductor operating at room temperature. An important milestone in this material research was the discovery, five years ago, ${ }^{1}$ of ferromagnetism in Mndoped $\mathrm{GaAs}^{2}$ with the Curie temperature $T_{c}=110 \mathrm{~K} .{ }^{1}$ In the $\mathrm{Ga}_{1-x} \mathrm{Mn}_{x}$ As diluted magnetic semiconductor (DMS) with $x \approx 1-10 \%$, Mn substituting for Ga provides a local moment $S=5 / 2$ and a delocalized hole. ${ }^{3,4}$ Ferromagnetic coupling between Mn moments is mediated by the itinerant holes via a kinetic-exchange interaction. ${ }^{5}$ Recent progress in lowtemperature molecular-beam epitaxy growth and postgrowth annealing techniques has led to the increase of the transition temperature in (Ga,Mn)As DMS's by nearly $50 \mathrm{~K} .{ }^{6}$ This success is attributed to a smaller concentration of carrier and moment compensating defects, especially interstitial $\mathrm{Mn}$, in the optimally annealed samples. ${ }^{7}$ The latest observations are consistent with an approximately linear dependence of $T_{c}$ on $x$ and hole Fermi wave vector predicted by theory. ${ }^{8-10}$

In this Rapid Communication we address theoretically the possibility of increasing $T_{c}$ in $(\mathrm{Ga}, \mathrm{Mn}) \mathrm{As}$ DMS's by a nonmagnetic acceptor codoping, namely, by introducing substitutional $\mathrm{C}_{\mathrm{As}}$ impurities. Our work is partly motivated by a recent experimental observation of a marked enhancement of the Curie temperature in Mn-implanted GaAs:C samples compared to the Mn-implanted undoped GaAs layers. ${ }^{11} \mathrm{We}$ focus on two key issues related to high-density carbon doping.

(i) The effect of $\mathrm{C}$ impurities on the density of states in the semiconductor valence band is assessed using the tightbinding/coherent-potential-approximation (TB/CPA) description of the disordered semiconductor.

(ii) The strength of the hole-mediated ferromagnetic Mn-Mn coupling is compared for systems with and without $\mathrm{C}$ codoping using the TB/CPA results and $T_{c}$ is then esti- mated from a model that combines the $\mathbf{k} \cdot \mathbf{p}$ Kohn-Luttinger description of the GaAs valence bands and a mean-field treatment of the kinetic-exchange coupling between Mn local moments and the band holes.

Before we start with the detailed description of the models we use and of the results let us remark that another important issue for hole codoping in (Ga,Mn)As DMS's is the change of the substitutional $\mathrm{Mn}_{\mathrm{Ga}}$ formation energy caused by the presence of additional non-magnetic acceptors. ${ }^{12}$ It is not our intention here to discuss this property in detail, primarily because systematic and quantitatively reliable calculations should be done using $a b$ inito, density-functional theory tools which are beyond the scope of this Rapid Communication. In the following paragraph, we make only few qualitative remarks, based on our TB/CPA total energies, that compare effects of $\mathrm{C}_{\mathrm{As}}$ and $\mathrm{Be}_{\mathrm{Ga}}{ }^{13}$ acceptors on substitutional $\mathrm{Mn}$ incorporation in (Ga,Mn)As DMS's.

The TB/CPA results suggest that Be acceptors substituting for the same element as $\mathrm{Mn}$, i.e., for $\mathrm{Ga}$, lead to a strong enhancement of the $\mathrm{Mn}_{\mathrm{Ga}}$ formation energy. At the same time, the formation energy of interstitial $\mathrm{Mn}$ is suppressed and, hence, an increasing fraction of $\mathrm{Mn}$ is incorporated in the form of interstitial donors or electrically neutral MnAs or Mn clusters. This scenario, which leads to a decrease rather than an increase of $T_{c}$, has recently been established by extensive experimental studies of Be codoped (Ga,Mn)As samples. ${ }^{13}$ On the other hand, the TB/CPA calculations indicate that $\mathrm{C}$ acceptors lead to a much weaker increase of the $\mathrm{Mn}_{\mathrm{Ga}}$ formation energy. We surmise that this property stems from the anomalous nature of $\mathrm{C}_{\mathrm{As}}$ which acts as an acceptor, yet has a larger Pauling's electronegativity than As. The difference between the effects of $\mathrm{Be}_{\mathrm{Ga}}$ and $\mathrm{C}_{\mathrm{As}}$ impurities on $\mathrm{Mn}_{\mathrm{Ga}}$ incorporation is further enhanced at high doping levels where the competition of $\mathrm{Be}$ and $\mathrm{Mn}$ for the same lattice site starts to play a role. 
The above qualitative analysis of formation energies suggests that $\mathrm{C}_{\mathrm{As}}$ codoping is favorable for achieving high Curie temperatures. Unlike typical acceptors, however, $\mathrm{C}$ has a very different atomic size and, as already mentioned, a large electronegativity compared to the atom it substitutes for. The crucial question, addressed in the following paragraphs, is then to what extent doping by several percent of $\mathrm{C}_{\mathrm{As}}$ changes the semiconductor band structure and whether the model of shallow acceptor carrier-induced ferromagnetism still applies in $(\mathrm{Ga}, \mathrm{Mn})(\mathrm{As}, \mathrm{C})$.

Our calculations of the electronic structure of GaAs in the presence of $\mathrm{Mn}_{\mathrm{Ga}}$ and $\mathrm{C}_{\mathrm{As}}$ impurities are done using the tight-binding version of the coherent-potential approximation (see, e.g., Ref. 14). The CPA, in contrast to supercell calculations, is well suited for the mixed crystals with low concentrations of randomly distributed impurities. It provides estimates for configurationally averaged density of states (DOS) and related quantities. The configurational averaging restores full translational symmetry of the lattice and makes it possible to decompose the DOS into contributions from specific points in the Brillouin zone. The spectral density $A(k, E)$ then includes a detailed information about the dispersion of the electronic states in the reciprocal space. In addition, the width of the peaks of $A(k, E)$ defines the scattering rate of the band quasiparticles in various parts of the Brillouin zone due to the impurities.

The parametrization of the TB Hamiltonian provides correct band gap for a pure GaAs crystal ${ }^{15}$ and an appropriate exchange splitting of the Mn $d$ states. Local changes of the crystal potential at both $\mathrm{Mn}$ and $\mathrm{C}$ impurities, represented by shifted atomic levels, are estimated using Ref. 16. Longrange tails of the impurity potentials, which become less important with increasing level of doping, are neglected. (Note that the Thomas-Fermi screening length is only $3-5 \AA$ for typical carrier densities ${ }^{17}$ i.e., comparable to the lattice constant.) Also lattice relaxation effects are neglected within the CPA. This is well justified for $\mathrm{Mn}_{\mathrm{Ga}}$ impurities ${ }^{18,19}$ but becomes a more important issue in the case of $\mathrm{C}_{\mathrm{As}}$ impurities. Previous density-functional studies found a $-0.6 \%$ relative change of the GaAs lattice constant in a 64-atom supercell with a single $\mathrm{C}_{\mathrm{As}}$ impurity. ${ }^{20}$ Although the lattice relaxation around $\mathrm{C}_{\mathrm{As}}$ may change our results quantitatively, we expect that the dominant effects on the band structure and on the spectral broadening arise from the different atomic levels of C compared to As which is readily accounted for in the TB scheme.

In Fig. 1 we plot the spin-polarized DOS in $\mathrm{Ga}_{1-x} \mathrm{Mn}_{x} \mathrm{As}_{1-y} \mathrm{C}_{y}$ with $x=y=4 \%$ together with local DOS on host (As) and impurity $\left(\mathrm{C}_{\mathrm{As}}\right)$ atoms. Despite the remarkable difference between the atomic levels of $\mathrm{As}$ and $\mathrm{C}$, $\Delta \varepsilon_{p} \approx-1 \mathrm{eV}$, the local DOS on $\mathrm{C}_{\mathrm{As}}$ sites (thin full line) near the valence-band edge does not differ much from the local DOS on the As sites (dashed line). (A still larger full width of the valence band than $\Delta \varepsilon_{p}$ may partly explain this.) As a result, the total DOS (thick full line) is only weakly affected by the presence of $\mathrm{C}$ in this spectral region. In particular, the spin splitting of $0.26 \mathrm{eV}$ of the valence-band edge, directly related to the kinetic-exchange parameter $J_{p d}$, is nearly the same in systems with and without $\mathrm{C}_{\mathrm{As}}$ impuri-

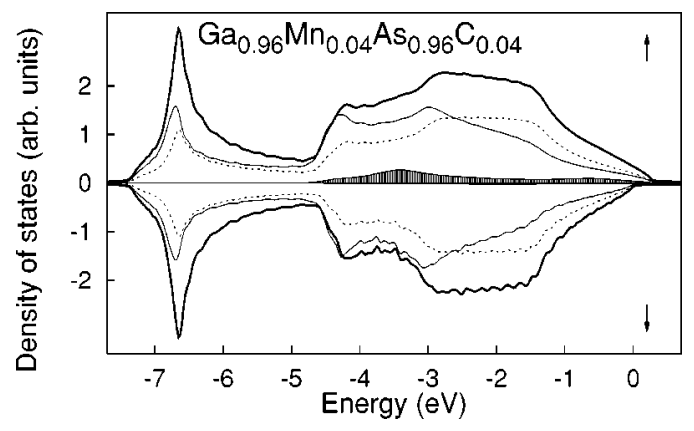

FIG. 1. TB/CPA density of states in $\mathrm{Ga}_{1-x} \mathrm{Mn}_{x} \mathrm{As}_{1-y} \mathrm{C}_{y}$ DMS with $x=y=4 \%$. Total DOS (thick full line), local DOS on host As (thin dashed line), and impurity $\mathrm{C}_{\mathrm{As}}$ (thin full line) atoms, and the DOS of Mn $d$ states are plotted as a function of energy, measured from the Fermi level.

ties. The shaded region in Fig. 1 shows the DOS of Mn $d$ states peaked near the energy of $-4 \mathrm{eV}$ which is consistent with results in $\mathrm{C}$ free $(\mathrm{Ga}, \mathrm{Mn}) \mathrm{As}^{21}$

It is important to point out that the substitution of $\mathrm{C}$ also has a very small effect on the linewidth of $A(k, E)$ for the states close to top of the valence band, i.e., electron scattering on $\mathrm{C}$ impurities does not disturb substantially the coherence of the Bloch states. This, together with an unchanged value of $J_{p d}$, implies that the additional disorder due to the codoping with $\mathrm{C}$ should not have any marked effect on the carrier mediated coupling between Mn moments. We check this more explicitly using the compatibility of the CPA with the Weiss mean-field theory. The strength of the Mn-Mn coupling is characterized by the energy cost of flipping one $\mathrm{Mn}_{\mathrm{Ga}}$ moment, which can be calculated for a given chemical composition. ${ }^{22}$ In Fig. 2, we plot this quantity as a function of the hole density $p$ for $x=y=4 \%$ (solid line) and $x=4 \%$ and $y=0$ (dashed line) samples. The hole density is varied in the calculations independently of $x$ and $y$, i.e., we assume implicitly a compensation whenever $p$ is smaller than the total density of $\mathrm{Mn}_{\mathrm{Ga}}$ and $\mathrm{C}_{\mathrm{As}}$ acceptors. The curves are nearly identical for systems with and without $\mathrm{C}$, as anticipated above. This means that, for a given hole concentration, the exchange coupling is quite insensitive to the large amount of the additional $\mathrm{C}_{\mathrm{As}}$ defects. We conclude our TB/ CPA considerations by stating that, as in the $\mathrm{Mn}_{\mathrm{Ga}}$ acceptor

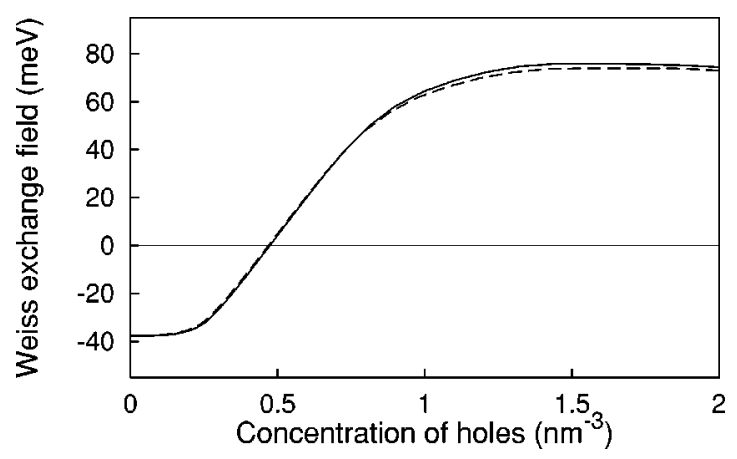

FIG. 2. Energy cost of flipping one $\mathrm{Mn}_{\mathrm{Ga}}$ moment, obtained from the TB/CPA spectra, is plotted as a function of the hole density for $x=y=4 \%$ (solid line), and $x=4 \%$ and $y=0$ (dashed line). 
case, the main effect of $\mathrm{C}_{\mathrm{As}}$ doping is a downward shift of Fermi energy with respect to the band edge, i.e., an increase of the number of holes in the valence band. In the following paragraphs we discuss prospects for ferromagnetic transition temperature enhancement by adding extra holes into the DMS valence band.

We use a model in which the itinerant holes are described by the GaAs host bands and the coupling to the local moments by a phenomenological constant $J_{p d}$ $=55 \mathrm{meV} \mathrm{nm}^{3}$. 5,23 This theory has been successful in describing semiquantitatively many non-trivial thermodynamic and transport properties of (Ga,Mn)As DMS's. ${ }^{9,10,24-28}$ In the simplest, virtual-crystal mean-field version of the model, which assumes ferromagnetic indirect coupling between $\mathrm{Mn}$ ions, the energy to flip the $\mathrm{Mn}_{\mathrm{Ga}}$ moment is proportional to the effective field $H_{e f f}=J_{p d}\langle s\rangle$. Here $\langle s\rangle$ is the mean spinpolarization density of the itinerant holes which increases with the hole density. This result is consistent with our TB/ CPA calculations, shown in Fig. 2, for hole densities not too much larger than the $\mathrm{Mn}_{\mathrm{Ga}}$ density. Recall that the concentration of the substitutional $\mathrm{Mn}$ ions is given by $N_{\mathrm{Mn}}$ $=4 x / a_{l c}^{3}$, where $a_{l c}$ is the GaAs lattice constant $\left(N_{\mathrm{Mn}}\right.$ $=0.88 \mathrm{~nm}^{-3}$ for $x=4 \%$, e.g.). For $p>N_{M n}$, RudermanKittel-Kasuya-Yosida (RKKY) oscillations of the Mn-Mn coupling ${ }^{5}$ start to play a role and the increasing number of antiferromagnetically coupled $\mathrm{Mn}$ moments leads to a saturation or even to a suppression of $T_{c}$, as seen in Fig. 2 for $p>1.3 \mathrm{~nm}^{-3}$. The mean-field theory that allows only for collinear ferromagnetic states is therefore likely to breakdown in this high hole-density region. Note also that for high $N_{\mathrm{Mn}}$ and low $p$, the direct antiferromagnetic Mn-Mn interaction takes over, as suggested by negative Weiss exchangefield values in Fig. 2, which sets another limit on the validity of the simple mean-field model. This low hole-density region is, however, not important for the high- $T_{c}(\mathrm{Ga}, \mathrm{Mn})(\mathrm{As}, \mathrm{C})$ DMS's we focus on in this paper.

We will now use the mean-field model to estimate the Curie temperature as a function of the $\mathrm{Mn}_{\mathrm{Ga}}$ local moment density and of the density of itinerant holes that can be varied independently. We emphasize that hole doping due to $\mathrm{C}$ is relevant also for systems with $p<N_{\mathrm{Mn}}$ since most of the experimental $(\mathrm{Ga}, \mathrm{Mn})$ As samples show some level of compensation, usually caused by interstitial Mn defects. In the calculations, the GaAs host band structure is obtained from the $\mathbf{k} \cdot \mathbf{p}$ Kohn-Luttinger model. ${ }^{24}$ We neglect the $\sim 10 \%-20 \%$ suppression of $T_{c}$ due to spin-wave fluctuations since the effect is compensated, to a large extent, by hole-hole exchange enhancement of $T_{c},{ }^{10}$ also neglected in the present calculations. Details of the model are described elsewhere $;, 3,10,24$ here we recall only the general $T_{c}$ expression we use $\mathrm{s}^{5,8,10}$

$$
k_{B} T_{c}=\frac{N_{\mathrm{Mn}} S(S+1)}{3} \frac{J_{p d}^{2} \chi}{\left(g \mu_{B}\right)^{2}}
$$

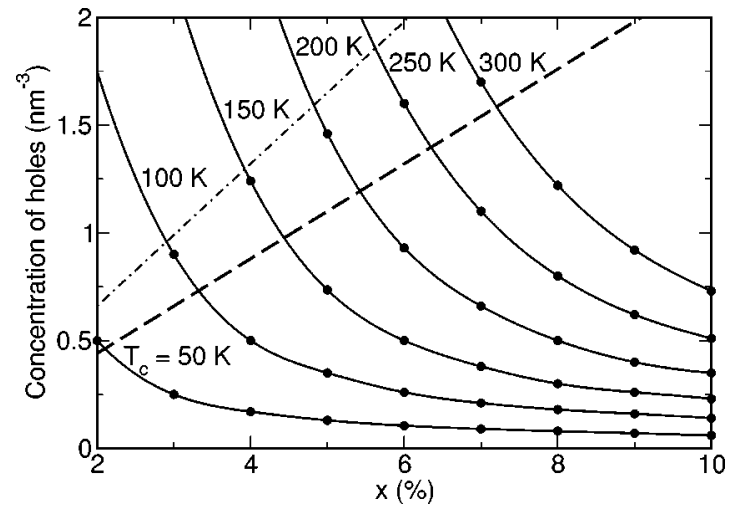

FIG. 3. Mean-field, k·p model calculations of constant $T_{c}$ curves are plotted in the $\mathrm{Mn}_{\mathrm{Ga}}$ doping $(x)$-hole-density $(p)$ diagram. Dashed line corresponds to $p=N_{\mathrm{Mn}}=4 x / a_{l c}^{3}$ and the dotteddashed line indicates approximately hole densities at which the mean-field model is expected to breakdown due to RKKY oscillations of the Mn-Mn indirect coupling.

where $\chi$ is the band-hole magnetic susceptibility which is roughly proportional to $p^{1 / 3}$. Figure 3 shows constant- $T_{c}$ curves calculated for critical temperatures ranging from $50 \mathrm{~K}$ to room temperature. The dashed line corresponds to $p$ $=N_{\mathrm{Mn}}$ and also indicates approximately the onset of RKKY oscillation effects on $T_{c}$. Note that the simple mean-field theory used to calculate the constant $-T_{c}$ curves is expected to breakdown above the dotted-dashed line where the large number of antiferromagnetically RKKY-coupled Mn moments might lead to a decrease rather than increase of $T_{c}$ with increasing hole density.

Figure 3 suggests that a substantial enhancement of the ferromagnetic transition temperature may be expected in hole codoped samples with high Mn moment concentration. Assuming, e.g., $x=10 \%$, an increase of the hole density from $p \approx 0.1 \mathrm{~nm}^{-3}$ to $p \approx 0.7 \mathrm{~nm}^{-3}$ leads to an increase of the theoretical $T_{c}$ from $50 \mathrm{~K}$ to $300 \mathrm{~K}$. For smaller $x$, a larger $\Delta p$ is needed to enhance $T_{c}$ by the same amount. Recent experiments have demonstrated that good quality $(\mathrm{Ga}, \mathrm{Mn}) \mathrm{As}$ DMS's can be grown with $x$ reaching $8 \% .{ }^{6}$ Based on the curves in Fig. 3 and our TB/CPA results we conclude that (Ga,Mn)As DMS's codoped with several percent of $\mathrm{C}$ should not be overlooked among potential candidates for a roomtemperature ferromagnetic semiconductor.

\section{ACKNOWLEDGMENTS}

We acknowledge fruitful discussions with Cammy Abernathy, Miroslav Cukr, Bryan Gallagher, Vít Novák, and Dan Park. This work was supported in part by the Welch Foundation, the Grant Agency of ASCR under Grant No. A1010214, the Grant Agency of the Czech Republic under Grant No. 202/02/0912, and DARPA/ONR under Grant No. N00014-00-095. 
${ }^{1}$ H. Ohno, Science 281, 951 (1998).

${ }^{2}$ H. Ohno, A. Shen, F. Matsukura, A. Oiwa, A. Endo, S. Katsumoto, and Y. Iye, Appl. Phys. Lett. 69, 363 (1996); J. De Boeck, R. Oesterholt, H. Bender, A. Van Esch, C. Bruynseraede, C. Van Hoof, and G. Borghs, J. Magn. Magn. Mater. 156, 148 (1996).

${ }^{3}$ J. König, J. Schliemann, T. Jungwirth, and A.H. MacDonald, in Electronic Structure and Magnetism of Complex Materials, edited by D.J. Singh and D.A. Papaconstantopoulos (SpringerVerlag, Berlin 2003), and references therein.

${ }^{4}$ T.C. Schulthess, Bull. Am. Phys. Soc. 48, 493 (2003); P.H. Dederichs, K. Sato, H. Katayama-Yoshida, and J. Kudrnovský, ibid. 48, 748 (2003).

${ }^{5}$ T. Dietl, Handbook of Semiconductors (North-Holland, New York, 1994), Vol. 3B; T. Dietl, A. Haury, and Y. Merle d'Aubigné, Phys. Rev. B 55, R3347 (1997).

${ }^{6}$ K.W. Edmonds, K.Y. Wang, R.P. Campion, A.C. Neumann, N.R.S. Farley, B.L. Gallagher, and C.T. Foxon, Appl. Phys. Lett. 81, 4991 (2002); K.C. Ku, S.J. Potashnik, R.F. Wang, M.J. Seong, E. Johnston-Halperin, R.C. Meyers, S.H. Chun, A. Mascarenhas, A.C. Gossard, D.D. Awschalom, P. Schiffer, and N. Samarth, ibid. 82, 2302 (2002); K.W. Edmonds, P. Boguslawski, K.Y. Wang, R.P. Campion, N.R.S. Farley, B.L. Gallagher, C.T. Foxon, M. Sawicki, T. Dietl, M.B. Nardelli, and J. Bernholc, cond-mat/0307140 (unpublished).

${ }^{7}$ K.M. Yu, W. Walukiewicz, T. Wojtowicz, I. Kuryliszyn, X. Liu, Y. Sasaki, and J.K. Furdyna, Phys. Rev. B 65, 201303 (2002).

${ }^{8}$ T. Jungwirth, W.A. Atkinson, B.H. Lee, and A.H. MacDonald, Phys. Rev. B 59, 9818 (1999).

${ }^{9}$ T. Dietl, H. Ohno, F. Matsukura, J. Cibert, and D. Ferrand, Science 287, 1019 (2000).

${ }^{10}$ T. Jungwirth, J. König, J. Sinova, J. Kučera, and A.H. MacDonald, Phys. Rev. B 66, 012402 (2002).

${ }^{11}$ Y.D. Park, J.D. Lim, K.S. Suh, S.B. Shim, J.S. Lee, C.R. Abernathy, S.J. Pearton, Y.S. Kim, Z.G. Khim, and R.G. Wilson, Phys. Rev. B 68, 085210 (2003).

${ }^{12}$ Although the formation energy is, strictly speaking, an equilibrium characteristic it is assumed that the basic features of its compositional dependence are reflected also in the dynamics of the nonequilibrium growth of the codoped materials.

${ }^{13}$ K.M. Yu, W. Walukiewicz, T. Wojtowicz, W.L. Lim, X. Liu, U. Bindley, M. Dobrowolska, and J.K. Furdyna, Phys. Rev. B 68, 041308(R) (2003).

${ }^{14}$ J. Mašek, B. Velický, and V. Janiš, J. Phys. C 20, 59 (1987).

${ }^{15}$ D.N. Talwar and C.S. Ting, Phys. Rev. B 25, 2660 (1982).
${ }^{16}$ W. Harrison, Electronic Structure and the Properties of Solid (Freeman, San Francisco, 1980).

${ }^{17}$ T. Jungwirth, M. Abolfath, Cairo Sinova, J. Kučera, and A.H. MacDonald, Appl. Phys. Lett. 81, 4029 (2002); M.P. LópezSancho and L. Brey, Phys. Rev. B 68, 113201 (2003).

${ }^{18}$ S. Mirbt, B. Sanyal, and P. Mphm, J. Phys.: Condens. Matter 14, 30130 (2002).

${ }^{19}$ J. Mašek, J. Kudrnovský, and F. Máca, Phys. Rev. B 67, 153203 (2003).

${ }^{20}$ C.D. Latham, R. Jones, S. Öberg, and P.R. Briddon, Phys. Rev. B 63, 155202 (2001).

${ }^{21}$ Our TB/CPA results, showing strongly localized $3 d$ Mn electrons and a dominantly delocalized nature of electrons near the Fermi energy, are consistent with recent density-functional calculations that account for correlations among $3 d$ Mn electrons [selfinteraction corrected local-density-approximation (LDA) or $\mathrm{LDA}+\mathrm{U}$ (Ref. 4)]. These band-structure theories are in an overall agreement with ferromagnetic resonance, photoemission, and transport measurements in metallic samples. An impurity band near the Fermi energy that appeared in previous densityfunctional calculations (see, e.g., Ref. 19 and S. Sanvito, G. Theurich, and N. Hill, J. Supercond. 15, 85 (2002), and references therein) is arguably an artefact of the density-functional schemes that omit strong correlations among the $3 d$ Mn electrons and, hence, underestimate the exchange splitting between occupied and empty $3 d \mathrm{Mn}$ states. This also leads to a substantial overestimate of the $J_{p d}$ parameter in these "uncorrected" density-functional theories.

${ }^{22}$ J. Mašek, Solid State Commun. 78, 351 (1991).

${ }^{23}$ H. Ohno, J. Magn. Magn. Mater. 200, 110 (1999); J. Okabayashi, A. Kimura, O. Rader, T. Mizokawa, A. Fujimori, T. Hayashi, and M. Tanaka, Phys. Rev. B 58, R4211 (1998).

${ }^{24}$ T. Dietl, H. Ohno, and F. Matsukura, Phys. Rev. B 63, 195205 (2001); M. Abolfath, T. Jungwirth, J. Brum, and A.H. MacDonald, ibid. 63, 054418 (2001).

${ }^{25}$ T. Dietl, J. König, and A.H. MacDonald, Phys. Rev. B 64, 241201 (2001).

${ }^{26}$ T. Jungwirth, Q. Niu, and A.H. MacDonald, Phys. Rev. Lett. 88, 207208 (2002).

${ }^{27}$ T. Jungwirth, Cairo Sinova, K.Y. Wang, K.W. Edmonds, R.P. Campion, B.L. Gallagher, C.T. Foxon, Q. Niu, and A.H. MacDonald, Appl. Phys. Lett. 83, 320 (2003).

${ }^{28}$ Jairo Sinova, T. Jungwirth, S.-R. Eric Yang, J. Kucera, and A.H. MacDonald, Phys. Rev. B 66, 041202 (2002). 